\title{
Software quality models: a comparative study
}

\begin{abstract}
In last decade, researchers have often tried to improve the usability, portability, integrity and other aspects of software in order for it to be more users friendly and gain user trust. Several approaches and techniques have been proposed to reduce the negative effects of software size and complexity. Moreover, several software quality models were proposed to evaluate general and specific type of software products. These models were proposed to evaluate general or specific scopes of software products. The proposed models were developed based on comparisons between the well-known models, in order to customize the closed model to the intended scope. These comparisons are leak of criteria that is conducted based on different perspectives and understanding. Therefore, a formal method of comparison between software quality models is proposed. The proposed method is applied on a comprehensive comparison between well-known software quality models. The result of the proposed method shows the strength and weaknesses of those models.
\end{abstract}

Keyword: Quality model; Model comparison; Model development 